Western University

Scholarship@Western

Physics and Astronomy Publications

Physics and Astronomy Department

2013

\title{
Periodic Arrays of Gold Nano-Disks Coupled with Evanescent Spectroscopy
}

Hao Jiang

Western University

Touraj Manifar

Western University

Aref Bakhtazad

Western University

Hossein Hojjati

Western University

Jayshri Sabarinathan

Western University

See next page for additional authors

Follow this and additional works at: https://ir.lib.uwo.ca/physicspub

Part of the Astrophysics and Astronomy Commons, and the Physics Commons

Citation of this paper:

Jiang, Hao; Manifar, Touraj; Bakhtazad, Aref; Hojjati, Hossein; Sabarinathan, Jayshri; and Mittler, Silvia, "Periodic Arrays of Gold Nano-Disks Coupled with Evanescent Spectroscopy" (2013). Physics and Astronomy Publications. 43.

https://ir.lib.uwo.ca/physicspub/43 
Authors

Hao Jiang, Touraj Manifar, Aref Bakhtazad, Hossein Hojjati, Jayshri Sabarinathan, and Silvia Mittler 
Journal of Applied Physics 113 (22) (2013) 224306

\title{
Periodic Arrays of Gold Nano-Disks Coupled with Evanescent Spectroscopy
}

\author{
Hao Jiang ${ }^{1}$, Touraj Manifar ${ }^{1,2}$, Aref Bakhtazad ${ }^{1}$, Hossein Hojjati ${ }^{1,2}$, Jayshri Sabarinathan ${ }^{1 a)}$ \\ and Silvia Mittler ${ }^{2 a}$ \\ ${ }^{1}$ Department of Electrical and Computer Engineering, Western University, \\ London, Ontario, N6A5B9, Canada. \\ ${ }^{2}$ Department of Physics and Astronomy, Western University, London, \\ Ontario, N6A3K7, Canada
}

\begin{abstract}
We study and analyze various spectral features of gold nano-disk arrays coupled with the evanescent field of an optical waveguide using finite difference time domain simulations for comparison to our previous work on this topic (Jiang et al, Journal of Lightwave Technology 27, no.13, (2009): 2264-2270). We find that the quadrupolar and dipolar peaks are both heavily dependent on the coherent interactions of the periodic array and show comparable performance for refractive index sensing applications. We also find sharp extinction peaks and dips in the simulated spectra, but they are not sensitive to the index change of the surface-bound layer. A detailed analysis on these sharp features reveals that the sharp extinction peaks are grating-induced quadrupolar modes and the dips represent the suppressed plasmon resonances caused by the photonic band gap.
\end{abstract}

\section{INTRODUCTION}

The extensive research interest in nanometer-dimension metallic nano-particles (NPs) has been mainly driven by their broad impact on the emerging disciplines of nanoengineering and nano-optics. Gold $(\mathrm{Au}) \mathrm{NPs}$, in particular, have been the focus of numerous investigations in recent years because of the promises offered by their optical, electronic, and chemical properties [1-4]. The unique optical properties are due to the localized surface plasmon resonance (LSPR) of the AuNPs, which is a collective a) Author to whom correspondence should be addressed. Electronic mail: jsabarin@uwo.ca or smittler@uwo.ca 
oscillation of the free electron gas confined in a nano-scale volume. The LSPR provides a remarkable enhancement on the evanescent electromagnetic field at the surface of the NPs, which has found important applications in surface-enhanced Raman spectroscopy [5, 6] and LSPR sensing [7-12].

By patterning the AuNPs into a periodic array, additional effects arise due to the interaction of the AuNPs with the photonic crystal (PC) lattice [13]. Such an interaction can result in unique optical properties useful for manipulating light at the nano-scale, and leads to strong local field enhancements. Meier et al. have theoretically studied the dipolar interactions of periodic arrays of NPs, and predicted the array effects on the plasmon peak and the radiative damping [14]. Lamprecht et al. first demonstrated experimentally the significant variations in the plasmon resonances of these periodic structures with lattice constants due to in-phase superposition of scattered light [15]. Linden et al. presented experimentally the selective suppression of the extinction of a periodic array of AuNPs on an indium tin oxide waveguide [16, 17]. The sharp gratinginduced plasmon mode of periodic arrays of metal NPs has been studied systematically both theoretically and experimentally [18-21]. AuNP arrays have been studied for refractive index sensing and showed great potential towards ultra-high sensing resolution [18, 19, 22-25]. Large-scale low-cost fabrication of the AuNP arrays has also been demonstrated recently [26].

Waveguide evanescent spectroscopy is based on the concept of exciting micro/nanoobjects positioned on a waveguide surface using the evanescent field of the waveguide and collecting the transmitted light [27]. Combining AuNPs with a waveguide can couple the LSPR with the evanescent field, providing interesting nano-optical phenomena, such as enhanced cross-talk due to proper polarization interrogation [27-29]. The biosensing approach based on AuNPs immobilized on the surface of waveguide has been studied $[30,31]$.

In our previous work, we have theoretically studied the AuNP-based photonic crystal on top of a slab waveguide for sensing applications [31]. The LSPR of the array is excited by the evanescent field of the waveguide mode and the wave-vector of the probing light lies in the same plane as the periodicities to benefit from the photonic crystal effects. The LSPR was found to greatly depend on both the periodicities and the waveguide dispersion. In this paper, we continue this theoretical study and explore further the various spectral features including dipolar peaks, quadrupolar peaks and sharp extinction peaks. We compare the sensing capabilities of those features and also briefly investigate the structure in experiments.

FIG. 1 schematically shows Au nano-disk array in a 2D lattice on top of a slab waveguide. The lattice constants along $\mathrm{X}$ and $\mathrm{Y}$ directions are given by $a_{x}$ and $a_{y}$, respectively. The 
nano-disks are $50 \mathrm{~nm}$ in height and slightly elliptical with diameters equal to $190 \mathrm{~nm}$ and $215 \mathrm{~nm}$ along the $\mathrm{X}$ and $\mathrm{Y}$ axis, respectively. The polarization of the TE and TM mode are also marked. The electric field oscillates only along the $\mathrm{Y}$ axis for TE modes and along both $\mathrm{X}$ and $\mathrm{Z}$ axis for TM modes.

\section{NUMERICAL SIMULATION CONFIGURATION}

The configuration of 3D finite difference time domain (FDTD) simulation is shown in FIG. 2, which is similar to the configuration previously used [31]. The structures of interest are 2D periodic arrays of Au nano-disks located on top of a waveguide with the periodic lattice in the X-Y plane. Periodic boundary conditions are applied in the $\mathrm{Y}$ direction, and the span of simulation region in the $\mathrm{Y}$ direction is equal to $a_{y}$ of the periodic array. This configuration is equivalent to simulating a periodic array infinitely extended in $\mathrm{Y}$ direction. Since the waveguide mode propagates from left to right, periodic boundaries could not be applied in the $\mathrm{X}$ direction due to the phase difference along the propagation path of the wave. Instead, perfectly matched layers (PMLs) are used as boundary condition, and 8 columns of AuNPs are assumed in the X direction to simulate the effects of periodicity in this direction. The waveguide consisted of a substrate with a refractive index $n_{\text {sub }}=1.55$, a $300 \mathrm{~nm}$ thick slab with a refractive index $n_{s}=1.65$ and the cover refractive index $n_{c}=1.33$ (water).

\section{RESULTS AND DISCUSSIONS}

Compared to the TM mode, the TE mode oscillates only along one direction and yields a simpler spectrum but it can excite all the physical features of interest. For the sake of brevity, we present and discuss the simulation results under excitation of fundamental TE mode, i.e., $\mathrm{TE}_{0}$ mode. The simulated spectra of changing lattice constant $a$ are shown in FIG. 3(a) and the dipolar peak, quadrupolar peak and sharp extinction peak features are marked clearly in FIG. 3(b). As the lattice constant increases, the dipolar peak red-shifts and the peak line width narrows, similar to those AuNP arrays under normal transmission $[14,15]$. The reduced line width can improve the sensing figure of merit (defined as sensitivity/line width) by a few times $[22,23]$. To understand these spectral features and their abilities in sensing, the spectrum for the structure of $a=500 \mathrm{~nm}$ without coating is additionally plotted as a black line in FIG. 3(b). Also shown is the spectrum with a $4 \mathrm{~nm}$ thick coating (red dashed line) with a refractive index of 1.44 encapsulating the Au nano-disks, mimicking biomolecules binding to the gold surface in an LSPR sensing application. Upon coating, the dipolar peak and the quadrupolar peak of the structure $a=500 \mathrm{~nm}$ both red-shift by about $5 \mathrm{~nm}$. 
The most interesting features in the simulated spectra are the sharp extinction peaks and dips, located very close to each other. In order to understand the origins of these features, the spectral position of the sharp extinction peaks and the dips are plotted versus the lattice constant in FIG. 4. The solid line is defined as $\lambda=a^{*} n_{\text {eff }}$, where $n_{\text {eff }}$ is the effective index of the $\mathrm{TE}_{0}$ mode. The waveguide configuration gives an effective index around 1.56 for the spectrum $700 \mathrm{~nm}-930 \mathrm{~nm}$. The sharp peak features all stay close to the solid line, which indicates the relation of these features to the waveguide mode. In order to examine these features closely, the mode profiles at the wavelength of the extinction peak and the extinction dip \#1 for the structure $a=500 \mathrm{~nm}$ were simulated and plotted as shown in FIG. 5 and FIG. 6. It was found, that, at the extinction peak wavelengths, the mode profile indicates a very strong enhancement of the electromagnetic fields of the quadrupolar mode in the periodic array. However, at the wavelength of the extinction dip, the plasmon resonance of the Au nano-disks is suppressed, without obvious quadrupolar mode features. The primary difference is that for the wavelength at the extinction peak, the anti-nodes of the electromagnetic field are located right under the Au nano-disks while for the wavelength of the extinction dip, the anti-nodes are located in the empty region between the Au nano-disks.

This phenomenon can be explained by considering the $2 \mathrm{D}$ periodic array as a superposition of a 1D photonic crystal in X direction and a 1D photonic crystal in $\mathrm{Y}$ direction. The Bragg reflection wavelength of a 1D photonic crystal structure is given by $\lambda_{b}=2 a^{*} n_{e f f} / m$, where $m$ is the grating order. In this case, since the lattice configuration is square, $a_{x}=a_{y}=a$, the second order Bragg reflection wavelength is given as $\lambda_{b}=a^{*} n_{\text {eff }}$. This wavelength actually corresponds to the second photonic band gap in the 1D photonic crystal [13]. For waves carrying wavelengths close to $\lambda_{b}$, the waves propagating in opposite directions, interfere with each other due to Bragg reflections, and the result is the formation of standing waves with two types of arrangements: one with the anti-nodes under the Au nano-disks which manifests itself in a sharp extinction peak and the other with the anti-nodes between the Au nano-disks which leads to a strong extinction dip. This extinction dip is similar to the selectively suppressed extinction reported from normal transmission of AuNP arrays on top of waveguides [16,17] and the sharp extinction peak is similar to the grating-induced mode reported from normal transmission of AuNP arrays [21].

We also found from the simulations, that, the simulated extinction peaks and dips are more affected by $a_{y}$ than $a_{x}$. As shown in FIG. 7, by simulating periodic arrays with unequal $a_{x}$ and $a_{y}$, one can see that the locations of the sharp peak and dip are basically dependent on $a_{y}$ only. In an additional simulation, we probed the structure with only one column of Au nano-disks in the $\mathrm{X}$ direction, i.e., a 1D array with periodicity only in the $\mathrm{Y}$ direction. The sharp extinction peak and dip still exist, but the amplitudes are much 
weaker. According to this comparison, the locations of the extinction peak and dip are strongly affected by the periodicity in $\mathrm{Y}$ direction while the periodicity in $\mathrm{X}$ direction only affects the amplitudes. We believe that $a_{x}$ and $a_{y}$ in reality should work equally. However, due to the simulation methods being used here, only a finite number of AuNPs could be simulated in X direction, which is very likely not sufficient to reveal the strong influence of $a_{x}$. In Y direction, the equivalent physical structure is an infinitely extended periodic array, which leads to a stronger coupling of the array. A similar phenomenon that the scattering line-shapes depend on the number of coupling nanoparticles can also be found from the discrete dipole approximation (DDA) calculations reported by Hicks et al. [20].

It should be pointed out, that the sharp extinction peaks reported here are of quadrupolar nature, which is different from the grating-induced modes caused by dipolar interactions reported by Felidj et al. [21]. Specifically, a dipolar resonator with a polarization in Y direction scatters light mainly along the $\mathrm{X}-\mathrm{Z}$ plane, and the grating-induced modes are thus more affected by $a_{x}$. For a quadrupolar resonator, light is scattered in more directions and the interactions of the periodic array are affected by periodicities in both dimensions. Due to the limited number of nano-disks in X direction in our study, the grating-induced mode in this case is basically determined by $a_{y}$. It can be expected that with larger number of periodicities in the $\mathrm{X}$ direction, a dependence of the grating-induced mode on both periodicities should be seen.

The sharp extinction peak and dip are located very close to each other, which might provide novel approaches to manipulate light. However, the sharp extinction peak and the dip were found to be not sensitive to the surface bound layer as shown in FIG. 3(b), meaning that these interesting spectral features are probably not appropriate for sensing applications.

We carried out experiments to study the Au nano-disk arrays on top of waveguides. The waveguides were fabricated using ion-exchange technique on BK7 glass substrates $[32,33]$ and $n_{\text {eff }}$ of the $\mathrm{TE}_{0}$ mode was tuned to match the simulated waveguide structure. The details of the experiment are presented in the supplemental information [34]. FIG. 8 shows the dipolar peaks excited by the $\mathrm{TE}_{0}$ mode. The experimental peaks follow the same trend as the simulations but the spectral positions are found at shorter wavelengths. Although $n_{\text {eff }}$ of the simulated and experimented waveguide are matched to each other, the experimental waveguide has a smaller refractive index on the top-most layer and caused the dipolar peaks to blue-shift. The experimental spectra also show seemingly sharp extinction peaks, however, such features cannot be verified yet due to the huge optical loss of the waveguides caused by silver particles formed during the ionexchange. 


\section{CONCLUSIONS}

We have studied and analyzed various spectral features of Au nano-disk arrays on top of waveguides using 3D FDTD simulations. Quadrupolar peaks and dipolar peaks show comparable performance for index sensing applications. The simulations revealed sharp extinction peaks and dips, very close to each other in the spectra. They are not sensitive to index changes of the surface-bound layer. We interpret that the sharp extinction peaks are grating-induced quadrupolar modes and the strong dips represent the suppressed plasmon resonance caused by the photonic band gap.

\section{ACKNOWLEDGMENT}

The authors acknowledge the funding from BIOPSYS, a strategic network funded by Natural Sciences and Engineering Research Council of Canada (NSERC). We would also like to acknowledge the funding of TEN program from Canadian Institute for Photonic Innovations (CIPI). We would like to thank Qiuquan Guo and Jun Yang in Department of Mechanical and Materials Engineering, University of Western Ontario for providing AFM investigations. The device fabrication was performed at the University of Western Ontario Nanofabrication Facility. The simulations were carried out using SHARCNET parallel computation facilities. We would also like to acknowledge the technical support on the 3D FDTD simulations from Lumerical Solutions Inc. Silvia Mittler also likes to acknowledge the Canadian government for the CRC program. 


\section{REFERENCES}

[1] S. Sun, C. B. Murray, D. Weller, L. Folks, and A. Moser, "Monodisperse FePt nanoparticles and ferromagnetic FePt nanocrystal superlattices," Science, vol. 287, no. 5460, pp. 1989-1992, 2000.

[2] C. J. Kiely, J. Fink, M. Brust, D. Bethell, and D. J. Schiffrin, "Spontaneous ordering of bimodal ensembles of nanoscopic gold clusters," Nature, vol. 396, pp. 444-446, 1998.

[3] D. I. Gittins, D. Bethell, D. J. Schiffrin, and R. J. Nichols, "A nanometre-scale electronic switch consisting of a metal cluster and redox-addressable groups," Nature, vol. 408, pp. 67-69, 2000.

[4] R. P. Andres, J. D. Bielefeld, J. I. Henderson, D. B. Janes, V. R. Kolagunta, C. P. Kubiak, W. J. Mahoney, and R. G. Osifchin, "Self-assembly of a two-dimensional superlattice of molecularly linked metal clusters," Science, vol. 273, no. 5282, pp. 16901693, 1996.

[5] C. L. Haynes, A. D. McFarland, and R. P. V. Duyne, "Surface-enhanced Raman spectroscopy," Analytical Chemistry, vol. 77, no. 17, pp. 338 A-346 A, 2005.

[6] C. T. Nguyen, J. T. Nguyen, S. Rutledge, J. Zhang, C. Wang, and G. C. Walker, "Detection of chronic lymphocytic leukemia cell surface markers using surface enhanced Raman scattering gold nanoparticles," Cancer Letters, vol. 292, pp. 91-97, 2010.

[7] K. A. Willets and R. P. Van Duyne, "Localized surface plasmon resonance spectroscopy and sensing," Annu. Rev. Phys. Chem., vol. 58, pp. 267-297, 2007.

[8] J. N. Anker, W. P. Hall, O. Lyandres, N. C. Shah, J. Zhao, and R. P. Van Duyne, "Biosensing with plasmonic nanosensors," Nature Materials, vol. 7, pp. 442-453, 2008.

[9] P. Englebienne, "Use of colloidal gold surface plasmon resonance peak shift to infer affinity constants from the interactions between protein antigens and antibodies specific for single or multiple epitopes," the Analyst, vol. 123, pp. 1599-1603, 1998.

[10] A. D. McFarland and R. P. Van Duyne, "Single silver nanoparticles as realtime optical sensors with zeptomole sensitivity," Nano Letters, vol. 3, no. 8, pp. 1057-1062, 2003.

[11] A. J. Haes and R. P. Van Duyne, "A nanoscale optical biosensor: Sensitivity and selectivity of an approach based on the localized surface plasmon resonance spectroscopy of triangular silver nanoparticles," Journal of the American Chemical Society, vol. 124, no. 35, pp. $10596-10604,2002$. 
[12] G. Raschke, S. Kowarik, T. Franzl, C. Sonnichsen, T. A. Klar, J. Feldmann, A. Nichtl, and K. Kurzinger, "Biomolecular recognition based on single gold nanoparticle light scattering," Nano Letters, vol. 3, no. 7, pp. 935-938, 2003.

[13] J. D. Joannopoulos, R. D. Meade, and J. N. Winn, Photonic Crystals: Molding the Flow of Light. Princeton University Press, 1995.

[14] M. Meier, A. Wokaun, and P. F. Liao, "Enhanced fields on rough surfaces: dipolar interactions among particles of sizes exceeding the Rayleigh limit," J. Opt. Soc. Am. B, vol. 2, no. 6, pp. 931-949, 1985.

[15] B. Lamprecht, G. Schider, R. T. Lechner, H. Ditlbacher, J. R. Krenn, A. Leitner, and F. R. Aussenegg, "Metal nanoparticle gratings: influence of dipoalr particle interaction on the plasmon resonance," Phys. Rev. Lett., vol. 84, no. 20, pp. 4721-4724, 2000.

[16] S. Linden, A. Christ, J. Kuhl, and H. Giessen, "Selective suppression of extinction within the plasmon resonance of gold nanoparticles," Appl. Phys. B, vol. 73, pp. 311-316, 2001.

[17] S. Linden, J. Kuhl, and H. Giessen, "Controlling the interaction between light and gold nanoparticles: selective suppression of extinction," Phys. Rev. Lett., vol. 86, no. 20, pp. 4688-4691, 2001.

[18] S. Zou, N. Janel, and G. C. Schatz, "Silver nanoparticle array structures that produce remarkably narrow plasmon lineshapes," J. Chem. Phys., vol. 120, no. 23, pp. 1087110875, 2004.

[19] S. Zou and G. C. Schatz, "Narrow plasmonic/photonic extinction and scattering line shapes for one and two dimensional silver nanoparticle arrays," J. Chem. Phys., vol. 121, no. 24, pp. 12606-12612, 2004.

[20] E. M. Hicks, S. Zou, G. C. Schatz, K. G. Spears, R. P. Van Duyne, L. Gunnarsson, T. Rindzevicius, B. Kasemo, and M. Kall, "Controlling plasmon line shapes through diffractive coupling in linear arrays of cylindrical nanoparticles fabricated by electron beam lithography," Nano Letters, vol. 5, no. 6, pp. 1065-1070, 2005.

[21] N. Felidj, G. Laurent, J. Aubard, G. Levi, A. Hohenau, J. R. Krenn, and F. R. Aussenegg, "Grating-induced plasmon mode in gold nanoparticle arrays," J. Chem. Phys., vol. 123, p. 221103, 2005.

[22] H. Jiang, J. Sabarinathan, and S. Mittler, "Effects of coherent interactions on the sensing characteristics of near-infrared gold nanorings," Journal of Physical Chemistry C, vol. 114, no. 36, pp. 15 243-15 250, 2010. 
[23] H. Jiang, T. Li, E. Ertorer, J. Yang, J. Sabarinathan, and S. Mittler. "A biosensor based on periodic arrays of gold nanodisks under normal transmission," Sensors and Actuators A: Physical, vol. 189, pp. 474-480, 2013

[24] V. Kravets, F. Schedin, A. Kabashin, and A. Grigorenko, "Sensitivity of collective plasmon modes of gold nanoresonators to local environment," Optics Letters, vol. 35, pp. 956-958, 2010.

[25] A. Nikitin, A. Kabashin, and H. Dallaporta, "Plasmonic resonances in diffractive arrays of gold nanoantennas: near and far field effects," Optics Express, vol. 20, pp. 27941-27952, 2012.

[26] E. Ertorer, F. Vasefi, J. Keshwah, M. Najiminaini, C. Halfpap, U. Langbein, J. JL Carson, D. W. Hamilton, and S. Mittler, "Large area periodic, systematically changing, multishape nanostructures by laser interference lithography and cell response to these topographies," Journal of Biomedical Optics, vol. 18, no. 3, pp. 035002, 2013.

[27] A. K. A. Aliganga, I. Lieberwirth, G. Glasser, A.-S. Duwez, Y. Sun, and S. Mittler, "Fabrication of equally oriented pancake shaped gold nanoparticles by SAM-templated OMCVD and their optical response," Organic Electronics, vol. 8, no. 2-3, pp. 161 - 174, 2007.

[28] P. Rooney, A. Rezaee, S. Xu, T. Manifar, A. Hassanzadeh, G. Podoprygorina, V. Bohmer, C. Rangan, and S. Mittler, "Control of surface plasmon resonances in dielectrically coated proximate gold nanoparticles immobilized on a substrate," Phys. Rev. $B$, vol. 77, p. 235446, 2008.

[29] S. Xu, G. Podoprygorina, V. Bohmer, Z. Ding, P. Rooney, C. Rangan, and S. Mittler, "Tetraurea calix[4]arenes with sulfur functions: synthesis, dimerization to capsules, and self-assembly on gold," Org. Biomol. Chem., vol. 5, pp. 558-568, 2007.

[30] S. Hashemi Rafsanjani, T. Cheng, S. Mittler, and C. Rangan, "A novel biosensing approach based on linear arrays of immobilized gold nanoparticles," Journal of Applied Physics, vol. 107, no. 7, p. 094303, 2010.

[31] H. Jiang, J. Sabarinathan, T. Manifar, and S. Mittler, "3D FDTD analysis of goldnanoparticle-based photonic crystal on slab waveguide," J. Lightwave Technol., vol. 27, no. 13, pp. 2264-2270, 2009.

[32] M. Weisser, F. Thoma, B. Menges, U. Langbein, and S. Mittler-Neher, "Fluorescence in ion exchanged BK7 glass slab waveguides and its use for scattering free loss measurements," Optics Communications, vol. 153, no. 1-3, pp. 27 - 31, 1998.

[33] A. Hassanzadeh, M. Nitsche, S. Armstrong, N. Nabavi, R. Harrison, S. J. Dixon, U. Langbein, and S. Mittler, "Optical waveguides formed by silver ion exchange in Schott 
SG11 glass for waveguide evanescent field fluorescence microscopy: evanescent images of HEK293 cells,” J. Biomed. Opt., vol. 15, p. 036018, 2010.

[34] See supplementary material as [URL will be inserted by AIP] for experimental details: sample fabrication and experimental set-up 


\section{FIGURE CAPTIONS}

FIG.1: Diagram of the structure configuration. A 2D periodic array of elliptical gold nanodisks is patterned on top of a waveguide. The LSPR is excited by the evanescent field of the waveguide mode. The polarization directions of the TE and the TM waveguide mode are shown with respect to the nano-disk geometry. The ellipticity of the nano-disks in the diagram has been shown slightly exaggerated for clarification.

FIG.2: 3D FDTD simulation configuration. Both top view and side view of the simulation configuration are presented. Periodic boundaries were applied in $\mathrm{Y}$ direction. PMLs boundaries were applied in $\mathrm{X}$ and $\mathrm{Z}$ directions. 8 columns of Au nano-disks were created along $\mathrm{X}$ direction to simulate the effects of periodicity in the $\mathrm{X}$ direction.

FIG.3: FDTD simulation results of a periodic array (square lattice) of Au nano-disks on top of a waveguide. (a) Simulated extinction spectra with changing lattice constant of a square lattice coupled to the $\mathrm{TE}_{0}$ mode. Lattice constants $a$ for each curve are given in the legend. (b) The peak features associated with the structure with lattice constant $a=500 \mathrm{~nm}$. The black line is the spectrum of the bare nano-disks while the dashed red line represents the spectrum of the nano-disks coated with a $4 \mathrm{~nm}$ shell with $n=1.44$. The important spectral features are marked on the spectrum. With the coating, the quadrupolar peak and dipolar peak red-shifted, but the sharp extinction peak and dips did not shift significantly.

FIG.4: Spectral positions of the sharp extinction peaks and dips in the extinction spectra. The solid line depicts the second order Bragg reflection wavelength.

FIG.5: The simulated mode profile at the wavelength of the sharp extinction peak for the device $a=500 \mathrm{~nm}$. The X-Y cut is the horizontal cut crossing the center of the nano-disks and the $\mathrm{X}-\mathrm{Z}$ cut is the vertical cut crossing the center of the nano-disks. 
FIG.6: The simulated mode profile at the wavelength of the extinction dip for the device $a$ $=500 \mathrm{~nm}$. The $\mathrm{X}-\mathrm{Y}$ cut is the horizontal cut crossing the center of the nano-disks and the $\mathrm{X}-\mathrm{Z}$ cut is the vertical cut crossing the center of the nano-disks.

FIG.7: Simulated extinction spectra of unequal lattice constant configurations. $a_{x}$ and $a_{y}$ of each curve are given in the legend. The lowest curve is obtained from a structure with only 1 column of gold nanodisks in the $\mathrm{X}$ direction, i.e. without any periodicity in $X$ direction. This curve represents the response of a 1D array, periodic in the $\mathrm{Y}$ direction. To show the data effectively, the data of this curve has been multiplied by a factor of 8 .

FIG.8: Dipolar peaks of the Au nano-disk arrays excited by a $\mathrm{TE}_{0}$ mode. The red circles and black squares represent simulation and experiment results, respectively. The solid line depicts the second order Bragg reflection wavelength.

Figures

FIG.1

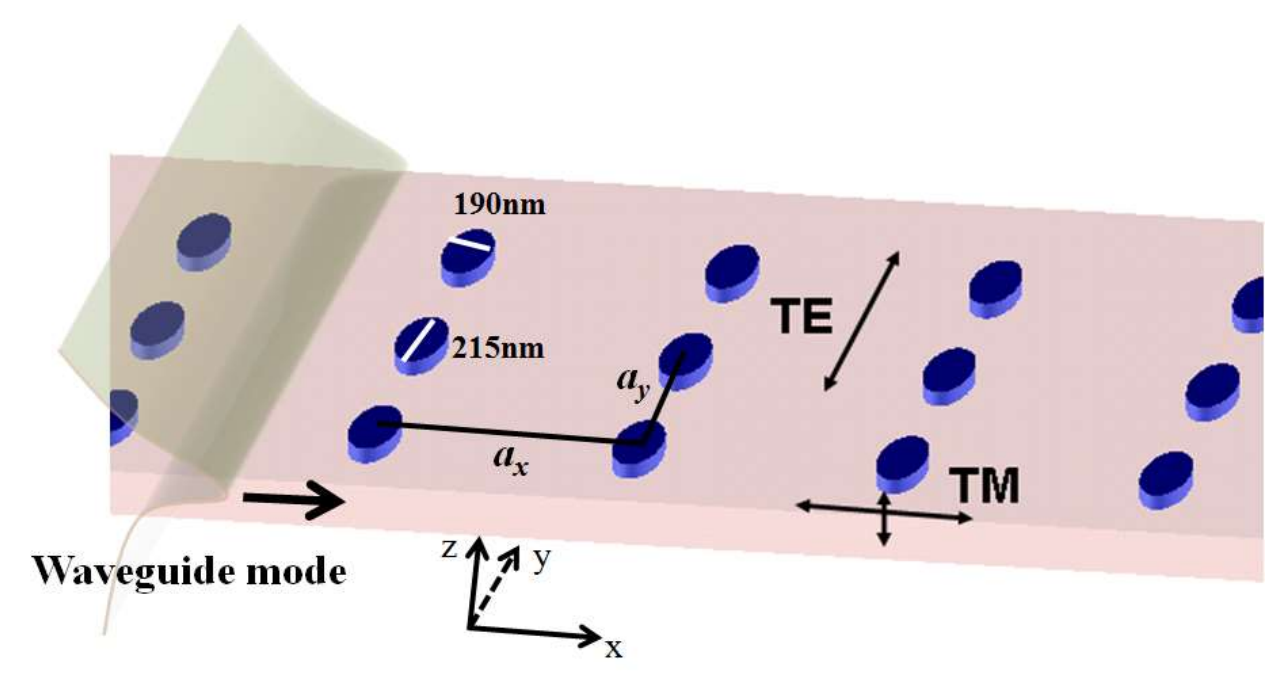




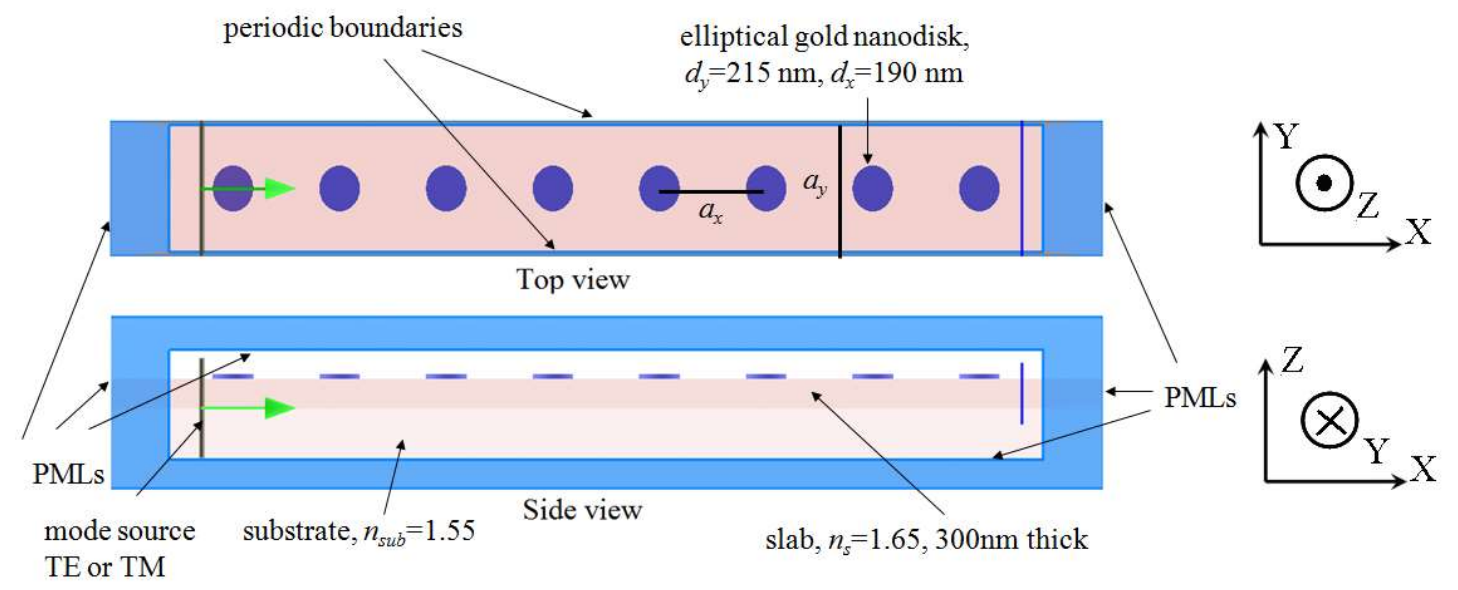

\section{FIG.3}
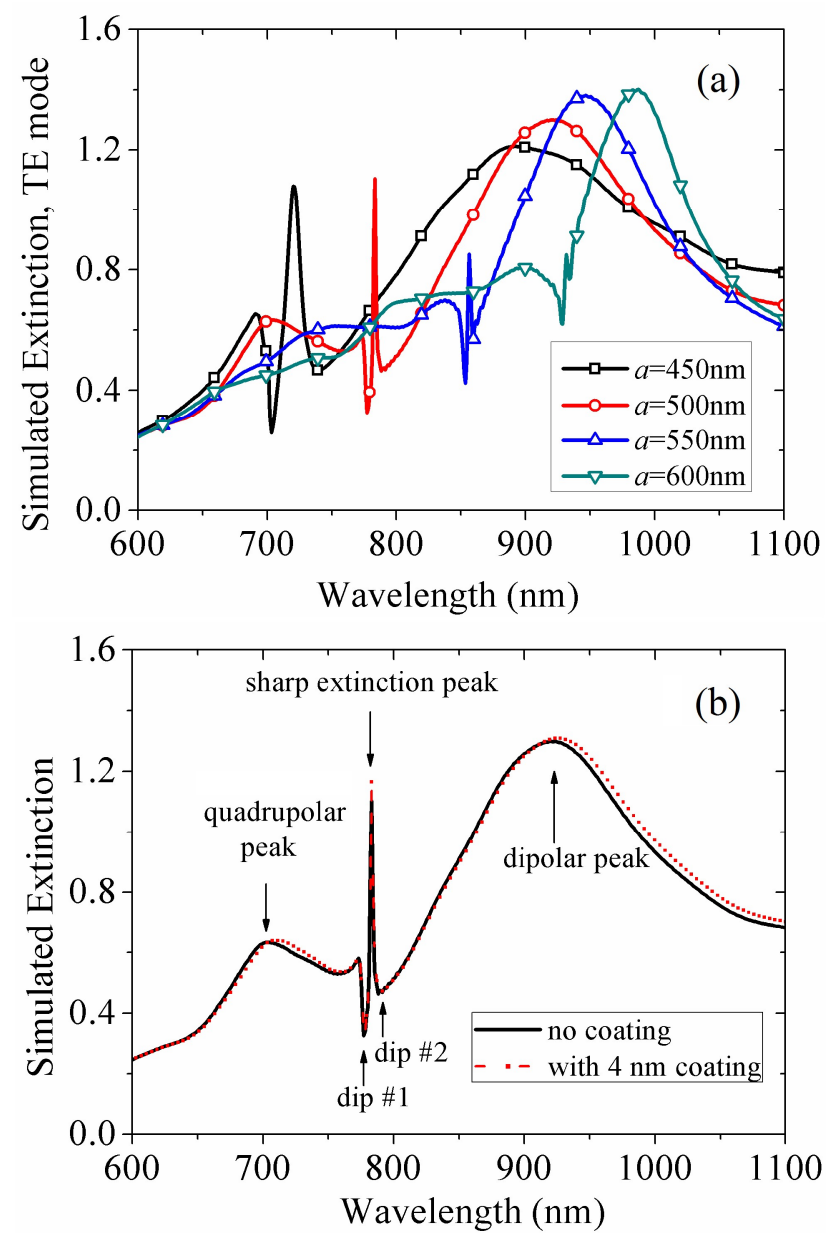
FIG.4

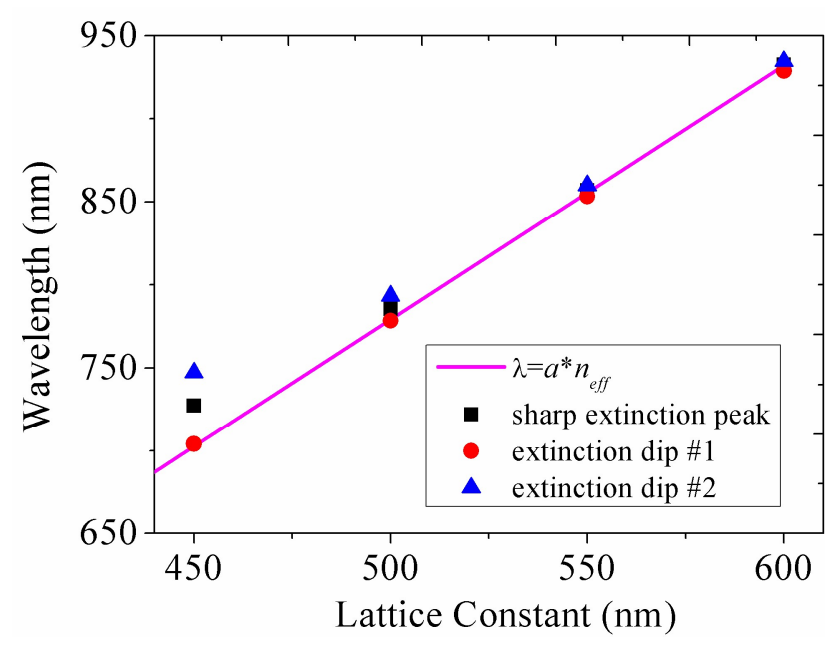

Fig. 5

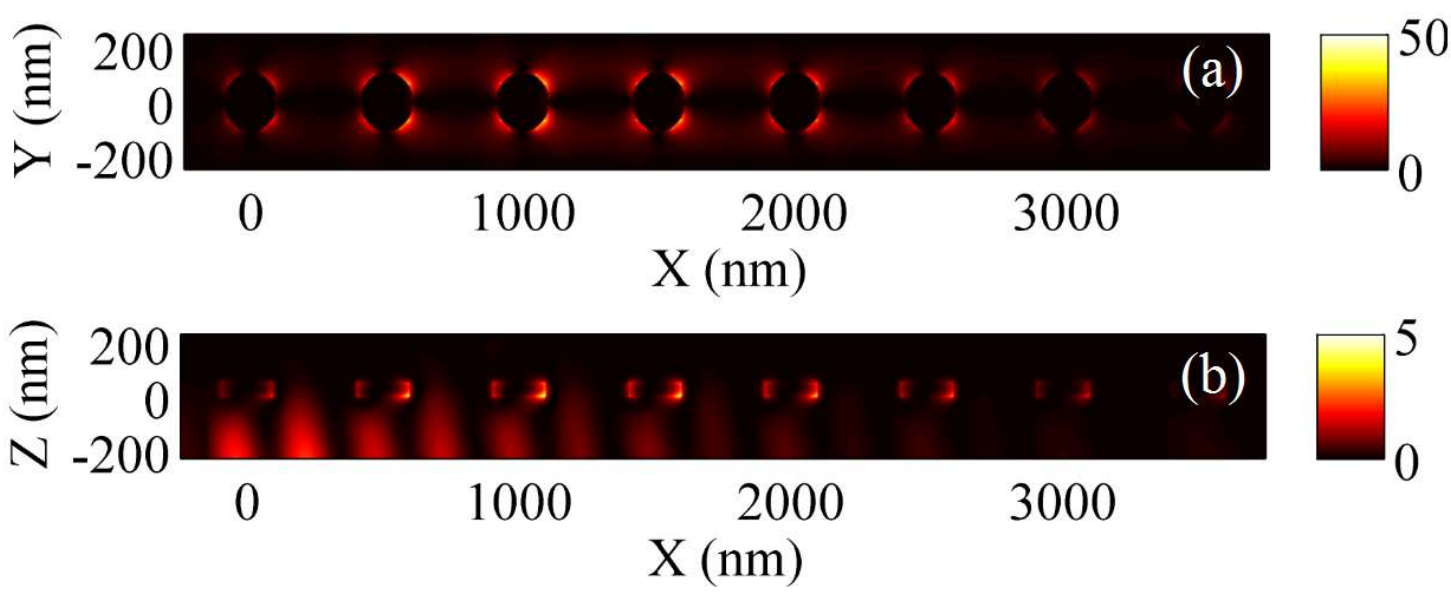


FIG.6

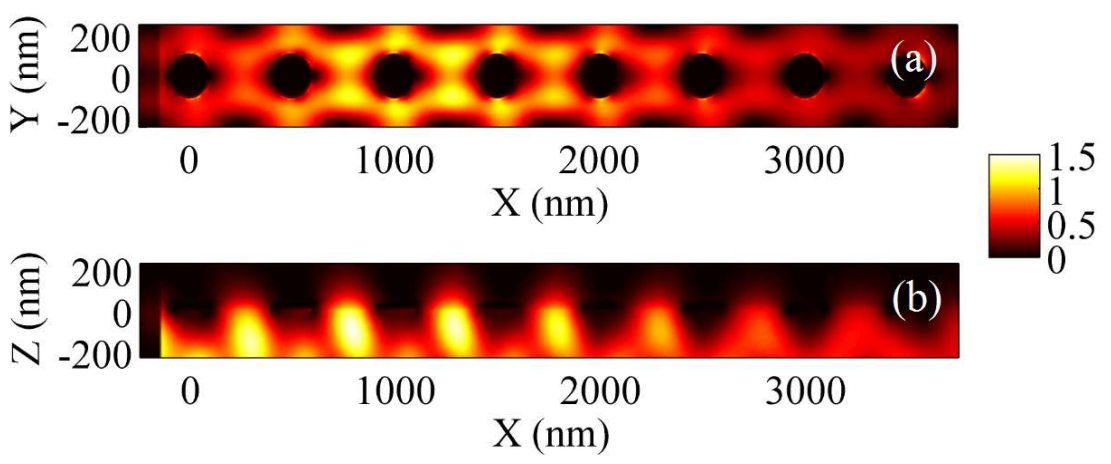

FIG.7

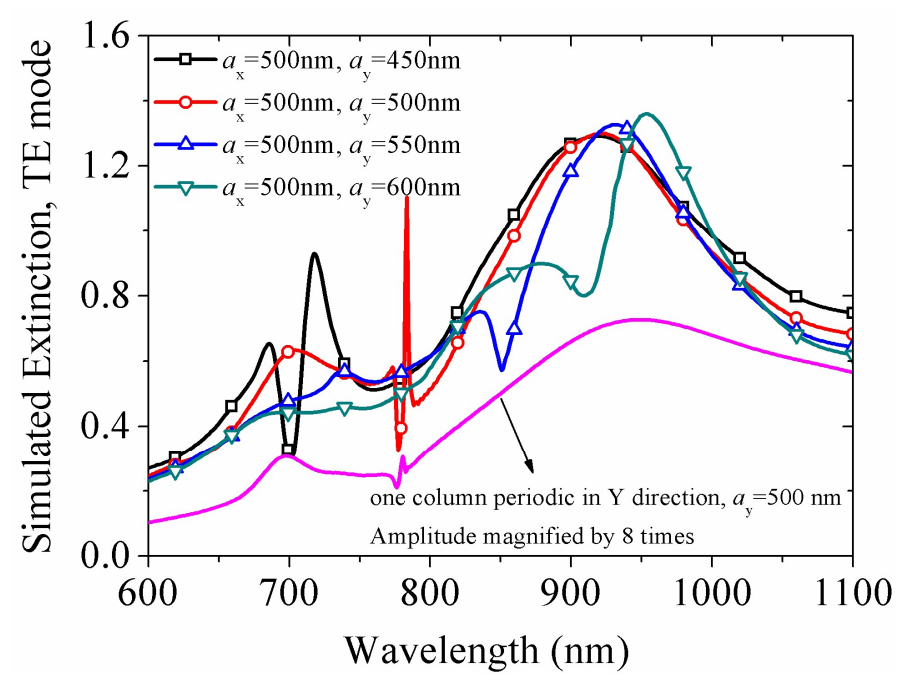

FIG.8

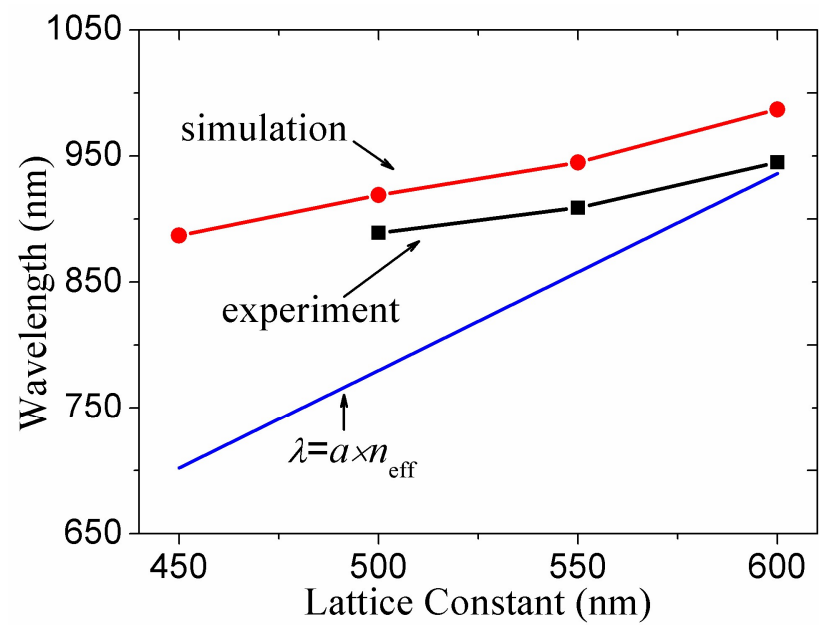


FIG.9 